\title{
TENSION IDENTIFICATION OF PIPELINE COMPENSATORS
}

\author{
Tadeusz SMOLNICKI $^{1}$, Pawel MAŚLAK ${ }^{2}$, Piotr ODYJAS $^{3}$, Jacek KARLIŃSKI ${ }^{4}$
}

1 Wroclaw University of Science and Technology, Faculty of Mechanical Engineering, Wybrzeże Wyspiańskiego 27, 50-370, Wroclaw, Poland. E-mail: tadeusz.smolnicki@pwr.edu.pl

2 Wroclaw University of Science and Technology, Faculty of Mechanical Engineering, Wybrzeże Wyspiańskiego 27, 50-370, Wroclaw, Poland. E-mail: pawel.maslak@pwr.edu.pl

3 Wroclaw University of Science and Technology, Faculty of Mechanical Engineering, Wybrzeże Wyspiańskiego 27, 50-370, Wroclaw, Poland. E-mail: piotr.odyjas@pwr.edu.pl

4 Wroclaw University of Science and Technology, Faculty of Mechanical Engineering, Wybrzeże Wyspiańskiego 27, 50-370, Wroclaw, Poland. E-mail: jacek.karlinski@pwr.edu.pl

\section{Introduction}

Process installations in the chemical industry often undergo very high thermal stresses, which cause significant changes in linear dimensions. Flexible slings and supports and compensators are used to limit the stresses caused by thermal phenomena. The basic problem in selecting a compensation circuit is the complex spatial state of the load occurring in the installation. The required rigidity of system components is determined solely on the basis of a simple linear model that does not take into account the complex form of the deformation of the compensators. This particularly applies to lens compensators (Figure 1.), which, in addition to the low axial stiffness, also have low stiffness and significant transverse and torsional stiffness. Stiffness characteristics are nonlinear and temperature dependent.

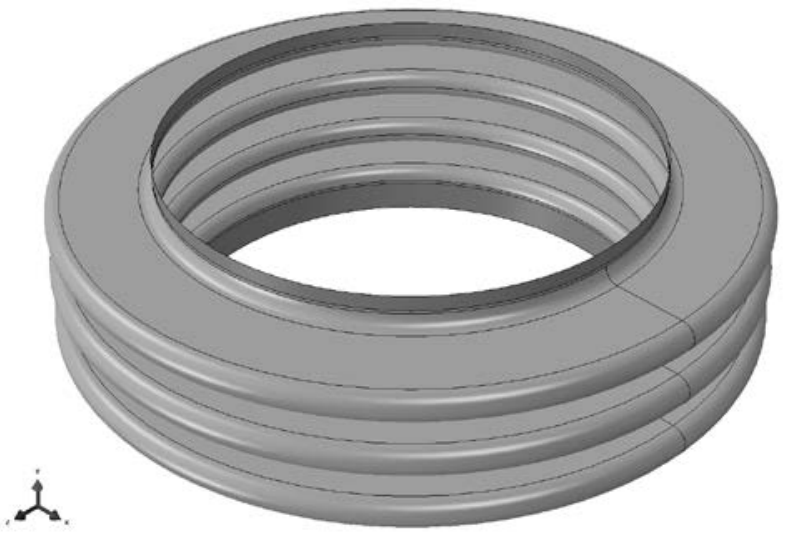

Fig. 1. 3-lens compensator

\section{Methodology of research}

Determining the state of the expansion of the compensators due to interactions between the individual pipelines requires the construction of computational models of the whole system (Figure 2). With such large models it is impossible to faithfully donate the geometry of the compensators [1].

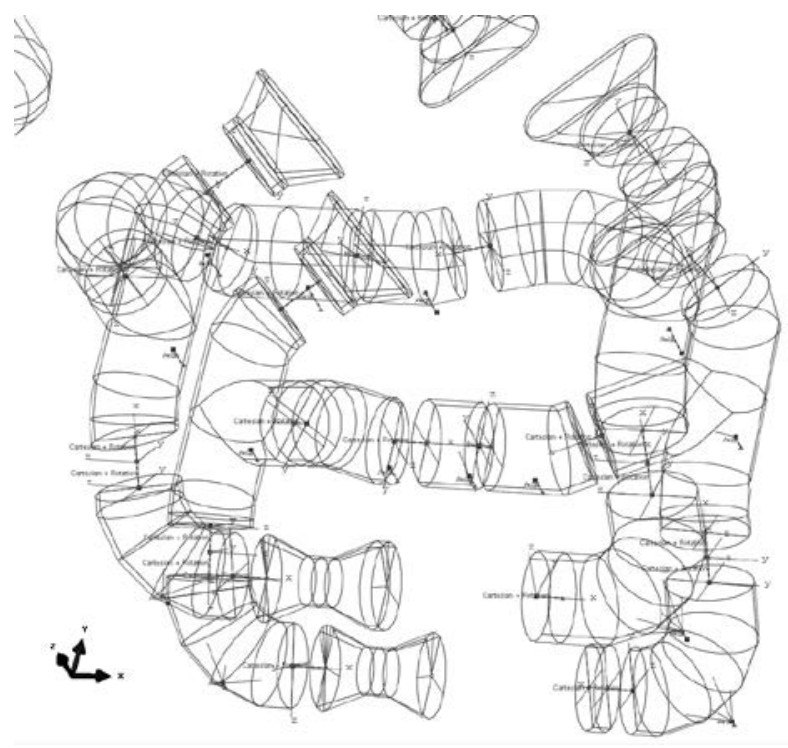

Fig. 2. Part of of the analyzed process installation

Therefore, it is necessary to conduct the analysis at different levels of detail (fig.3) [2,3].

The first step is to determine the stiffness matrix of the substitute element of compensators.

In the second step, substitute elements are applied to the global model of the whole system. After thermal-displacement analysis, the displacement of the connector points of the 


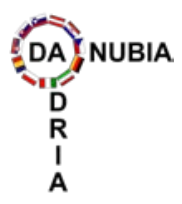

34th Danubia-Adria Symposium on Advances in Experimental Mechanics

University of Trieste, Italy, 2017 connectors to the local models and the further strength and fatigue analysis are extrapolated.

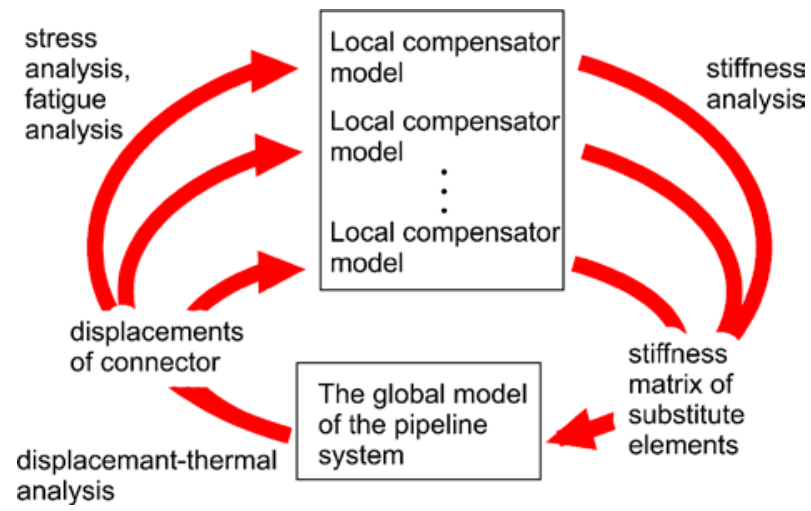

Fig. 3. Methodology.

\section{Determination of boundary conditions}

Boundary conditions for global model analysis are measured long-term during system operation. These are the temperature of the flowing medium and the pressure. Data acquisition time was 2 months. Because of the aggressiveness of the environment and the thermal insulation, it was impossible to measure displacements. Additional load is gravity. Figure 4 shows an example of the temperature waveform recorded for $48 \mathrm{~h}$.

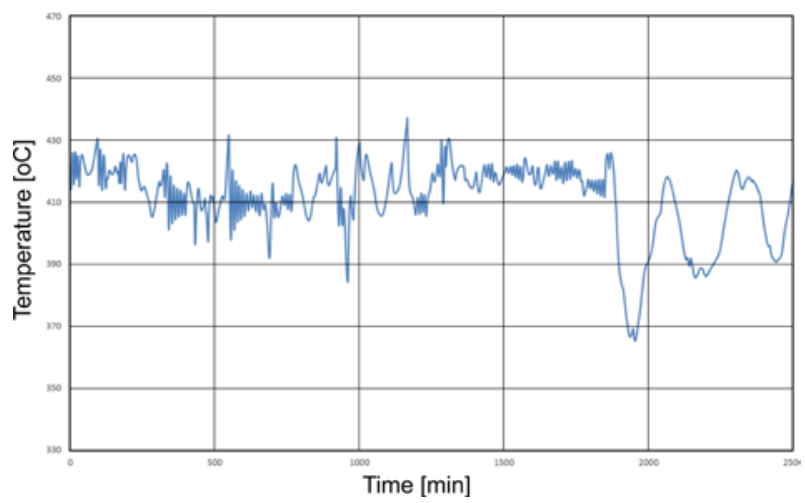

Fig. 4. Temperature waveform.

\section{Summary}

The purpose of the calculations was to check the correct choice of compensators. The methodology used was based on analysis at two different levels of model detailing. The average stresses and the amplitude of the stresses in the compensators were determined. It has been shown that some of the expansion joints mainly work on bending, ie, they mainly compensate the angular displacement, although their selection is based on the assumption of linear compensation. Further analysis will be based on the analysis of the variability rainflow stress in a long time operation on the basis of the waveforms recorded actual operating parameters to estimate fatigue life.

\section{References}

[1] Rusiński E., Czmochowski J., Smolnicki T.: Zaawansowana metoda elementów skończonych w konstrukcjach nośnych. Oficyna Wydaw. PWroc., 2000

[2] Smolnicki T., Karliński Jacek, Derlukiewicz D.: Identification of internal stresses in bolted flanged joints. Solid State Phenomena. 2010, vol. 165 , s. $353-358$

[3] Smolnicki T., Rusiński E., Karliński J.: FEM modelling of fatigue loaded bolted flange joints. Journal of Achievements in Materials and Manufacturing Engineering. 2007, vol. 22, iss. 1 , s. 69-72 